\title{
Influence of patient gender on admission to intensive care
}

\author{
R Raine, C Goldfrad, K Rowan, N Black
}

J Epidemiol Community Health 2002;56:418-423

See end of article for authors' affiliations

....................

Correspondence to: Dr R Raine, Health Services Research Unit, London School of Hygiene and Tropical Medicine, Keppel Street, London WCIE 7HT rosalind.raine@Ishtm.ac.uk

Accepted for publication 6 July 2001

\begin{abstract}
Study objectives: To compare the case mix and outcomes of male and female patients admitted to intensive care units for a wide range of conditions.

Design: Cross sectional study of prospectively collected data.

Setting: The Intensive Care National Audit and Research Centre, (ICNARC) Case Mix Programme.

Participants: 46587 admissions to 91 units across England, Wales, and Northern Ireland.

Main results: No gender differences were found in case mix on admission or in mortality for five conditions (cardiac arrhythmia, chronic obstructive airways disease, asthma, self poisoning, and seizures). There was some evidence of horizontal and vertical inequity for female patients with myocardial infarction and with neurological bleeding. Vertical equity was not achieved for male pneumonia and ventricular failure patients and for women with primary brain injury.

Conclusions: This study demonstrated, for the first time, possible inequitable use of intensive care for patients with certain conditions. This may be secondary to gender bias and can result in either overtreatment in the favoured group, or under-treatment in the neglected group. It would therefore be pertinent to re-examine these findings using other databases, and to further investigate the causative factors, including gender bias.
\end{abstract}

ntensive care medicine is concerned predominantly with the management of patients with acute, life threatening conditions in a specialised unit. ${ }^{12}$ It is assumed that admission to intensive care units (ICUs) is determined by the clinical needs of the patient rather than their sociodemographic characteristics. Studies in other areas of specialist care however, suggest that a patient's sex can influence health care utilisation. ${ }^{3}$ Is the same true for intensive care? Does any "gender bias" exist in the decision to admit patients to an ICU?

Examination of differences in case mix (age, acute severity, comorbidity, reason for admission) between male and female admissions to ICU allows the extent to which horizontal equity (equal use for equal need) exists across a range of conditions. If women are consistently found to have significantly more severe disease at admission than men, this would suggest that a higher clinical threshold for admission operates for women-that is, horizontal inequity. If such inequity does exist, it may be attributable to clinicians' views of "gender norms" - a hypothesis that suggests that conditions that are perceived as affecting predominantly one sex lead to patients of that sex being less severely ill than the other sex on admission to ICU. For example, the stereotypical myocardial infarction patient is male, so men with this condition would be less severely ill than women. ${ }^{4}$ In contrast, women with asthma would be less severe because the prevalence and mortality from asthma is higher in women. ${ }^{5}$

Vertical equity also needs to be assessed. ${ }^{6}$ Vertical equity refers to the concept that people with greater clinical needs should have more treatment than those with lesser needs (unequal use for unequal need). Thus, patients with severe disease should be more likely to be admitted to ICU compared with patients with a milder form of the same disease, regardless of gender. If gender differences in illness severity at admission exist, then vertical equity would be achieved if unequal use of health care was accompanied by no gender differences in risk adjusted mortality. In such a situation horizontal inequity can be justified. Conversely, if one sex exhibited both more severe disease at admission and higher risk adjusted hospital mortality, then this would suggest their greater need for care was not being met, and, thus vertical inequity existed.

The first objective of this study was to assess whether horizontal inequity on the basis of gender exists in the UK National Health Service, and if so, the extent to which views of "gender norms" can explain it. The second objective was to explore whether or not vertical equity exists.

\section{METHODS}

Data collection

The Intensive Care National Audit and Research Centre (ICNARC) Case Mix Programme (CMP) is a national comparative audit of patient outcome from both intensive care and high dependency units. ${ }^{78}$ Data are collected prospectively by trained data collectors according to carefully constructed, defined rules and definitions. ${ }^{9}$ The data had undergone extensive, external data validation before incorporation into the CMP database. At the time of these analyses data from 91 voluntarily contributing units across England, Wales, and Northern Ireland on 46587 admissions were available. The data were collected over three years.

The APACHE II (Acute Physiology and Chronic Health Evaluation) method was used to produce an objective summary score to describe the illness severity of each intensive care patient. ${ }^{10}$ This score takes account of age, acute illness severity in the first 24 hours in ICU (referred to as the acute physiology score), chronic illness severity, the reason for admission, and whether or not the patient had undergone emergency surgery immediately prior to admission to ICU (box l).

\section{Identification of clinical categories for analysis}

The criteria for selecting categories for analysis were that they should be a frequent primary cause of admission (at least 500 cases). By limiting selection to primary reasons for admission, this ensured that patients with comorbidity that included conditions studied, would not be selected twice. Selection was also influenced by our need to include some 
Box 1 Variables incorporated in the APACHE II method

Age

Acute physiology

- Temperature

- Blood pressure

- Heart rate

- Respiratory rate

- Arterial blood gas

- Arterial pH

- (Serum bicarbonate: use if no arterial pH)

- Serum sodium

- Serum potassium

- Serum creatinine

- Packed cell volume

- White blood cell count

- Glasgow Coma Score

Past medical history

(defined by the presence of one or more of 16 defined conditions/therapies: biopsy proven cirrhosis, portal hypertension, hepatic encephalopathy, very severe cardiovascular disease, severe respiratory disease, AIDS, metastatic disease, acute myelogenous leukaemia or acute lymphocytic leukaemia or multiple myeloma, chronic myelogenous leukaemia or chronic lymphocytic leukaemia, lymphoma, congenital immunohumoral or cellular immune deficiency state, home ventilation, chronic renal replacement therapy, corticosteroid treatment, radiotherapy, chemotherapy).

Surgical status (post emergency status or not)

Reason for admission to intensive care unit

Vital status at ultimate discharge from hospital

conditions that occur more in one sex than the other. Surgical admissions defined as those admitted to ICU directly from theatre and/or recovery) were excluded because the decision to admit is partly a preoperative clinical decision and, therefore, partly influenced by the illness severity of the patient before operating rather than at the time of admission. The only other exclusions were admissions aged less than 16 years or whose length of stay in the unit was less than eight hours.

Ten categories were identified. For five categories, sufficient cases were available to allow a discrete condition to be analysed (acute myocardial infarction, asthma, chronic obstructive airways disease, pneumonia, self poisoning). Acute myocardial infarction and chronic obstructive airways disease are more prevalent in men than women whereas asthma and self poisoning are more prevalent in women. ${ }^{111}{ }^{12}$ The other five categories included more than a single discrete condition: seizures, primary brain injury, ventricular failure, cardiac arrhythmias, and neurological bleeding. Diagnoses were only grouped into categories if they shared the same APACHE II diagnostic coefficient. This coefficient was previously derived by modelling data from 26 ICUs in the UK and Ireland. ${ }^{10}$ This was necessary because patients with the same APACHE II score may have different probabilities of hospital death depending on their diagnosis.

\section{Analysis}

Thresholds for admission in terms of age, past medical history, acute physiology score (comprising the 12 physiology variables collected for the APACHE II score), and APACHE II score were calculated for men and women for each category. The distribution of ages, acute physiology, and APACHE II scores sometimes had an asymmetrical frequency distribution with most patients having low scores. Therefore, both mean and median values were calculated. Both the $t$ test for independent samples and the Mann-Whitney $U$ test were used to compare the distribution of continuous variables. The Pearson $\chi^{2}$ test was used to compare categorical variables.

Case mix adjusted hospital mortality (defined as death during hospital stay, regardless of length of stay) for men and for women were compared for each of the 10 categories. This was done by calculating odds ratios for hospital mortality in men compared with women before and after adjusting for APACHE II score.

The power to detect a clinically significant difference in case mix on admission to ICU (where one really existed) with 95\% precision using the sample sizes available was examined retrospectively and is reported below. It was assumed that the minimum difference in APACHE II scores between men and women likely to be clinically important was $10 \%$.

\section{RESULTS}

The number of patients in each category and the probability of getting a statistically significant result $(p=0.05)$ with this sample if a true difference of $10 \%$ existed in APACHE II scores between men and women is shown in table 1. Apart from asthma and primary brain injury, the sample sizes provided adequate power.

\section{Acute myocardial infarction}

Male patients were significantly younger, fewer had a past medical history, and the APACHE II score was significantly lower than female patients (table 2). Men also had a lower hospital mortality than women, a difference that persisted after risk adjustment, though no longer significant at the 5\% level (adjusted OR $0.91,95 \%$ CI 0.65 to 1.28 ).

\section{Pneumonia}

There were no significant gender differences with respect to age, acute physiology score, past medical history, or APACHE II

Table 1 Number of patients in each category and probability of getting a significant result

\begin{tabular}{lrrr}
\hline Category & $\begin{array}{l}\text { Number (\%) } \\
\text { of men }\end{array}$ & $\begin{array}{l}\text { Number (\%) } \\
\text { of women }\end{array}$ & $\begin{array}{l}\text { Probability of achieving } \\
\text { significant result } \\
(p=0.05)(\%)\end{array}$ \\
\hline Acute myocardial infarction & $845(66.6)$ & $423(33.4)$ & 79 \\
Ventricular failure & $499(57.3)$ & $372(42.7)$ & 91 \\
Cardiac arrhythmia & $634(64.3)$ & $352(35.7)$ & 85 \\
Pneumonia & $1711(60.1)$ & $1136(39.9)$ & 100 \\
Chronic obstructive airways disease & $494(52.1)$ & $454(47.9)$ & 86 \\
Asthma & $233(32.2)$ & $490(67.8)$ & 27 \\
Self poisoning & $826(51.2)$ & $786(48.8)$ & 83 \\
Primary brain injury & $767(77.6)$ & $221(22.4)$ & 30 \\
Seizures & $326(54.5)$ & $272(45.5)$ & 66 \\
Neurological bleed & $480(55.6)$ & $383(44.4)$ & 76 \\
\hline
\end{tabular}




\begin{tabular}{|c|c|c|c|c|}
\hline Category & Measure & Men & Women & Difference \\
\hline Acute myocardial infarction & $\begin{array}{l}\text { Mean (median) age } \\
\% \text { PMH } \\
\text { Mean (median) APS } \\
\text { Mean (median) APACHE II } \\
\% \text { Hospital mortality } \\
\text { male: female crude odds ratio }(95 \% \mathrm{CI}) \\
\text { male: female adjusted odds ratio }(95 \% \mathrm{CI})\end{array}$ & $\begin{array}{l}64.2(66.0) \\
7.8 \\
11.8(10.0) \\
16.0(14.0) \\
46.4\end{array}$ & $\begin{array}{l}68.3(70.0) \\
13.8 \\
12.7(11.0) \\
17.8(16.0) \\
53.8 \\
0.71(0.54 \text { to } 0.93) \\
0.91(0.65 \text { to } 1.28)\end{array}$ & $\begin{array}{l}<0.001 \dagger \\
0.001^{*} \\
0.07 \dagger \\
0.002 \dagger \\
0.011^{*}\end{array}$ \\
\hline Pneumonia & $\begin{array}{l}\text { Mean (median) age } \\
\% \text { PMH } \\
\text { Mean (median) APS } \\
\text { Mean (median) APACHE II } \\
\% \text { Hospital mortality } \\
\text { male: female crude odds ratio }(95 \% \mathrm{CI}) \\
\text { male: female adjusted odds ratio }(95 \% \mathrm{CI})\end{array}$ & $\begin{array}{l}59.3(64.0) \\
24.0 \\
15.1(14.0) \\
19.7(19.0) \\
53.2\end{array}$ & $\begin{array}{l}58.1(63.0) \\
22.4 \\
15.1(15.0) \\
19.6(19.0) \\
48.2 \\
1.27(1.06 \text { to } 1.52) \\
1.28(1.06 \text { to } 1.54)\end{array}$ & $\begin{array}{l}0.08 \dagger \\
0.33^{*} \\
0.75 \dagger \\
0.75 \dagger \\
0.01^{*}\end{array}$ \\
\hline Chronic obstructive airways disease & $\begin{array}{l}\text { Mean (median) age } \\
\% \text { PMH } \\
\text { Mean (median) APS } \\
\text { Mean (median) APACHE II } \\
\% \text { Hospital mortality } \\
\text { male: female crude odds ratio }(95 \% \mathrm{CI}) \\
\text { male: female adjusted odds ratio }(95 \% \mathrm{CI})\end{array}$ & $\begin{array}{l}66.2(68.0) \\
41.0 \\
12.8(12.0) \\
19.1(18.0) \\
40.3\end{array}$ & $\begin{array}{l}65.4(67.0) \\
40.6 \\
12.3(12.0) \\
18.5(18.0) \\
36.7 \\
1.14(0.84 \text { to } 1.54) \\
1.05(0.77 \text { to } 1.43)\end{array}$ & $\begin{array}{l}0.23 \dagger \\
0.89^{*} \\
0.14 \dagger \\
0.15 \dagger \\
0.27 \dagger\end{array}$ \\
\hline Asthma & $\begin{array}{l}\text { Mean (median) age } \\
\% \text { PMH } \\
\text { Mean (median) APS } \\
\text { Mean (median) APACHE II } \\
\% \text { Hospital mortality } \\
\text { male: female crude odds ratio }(95 \% \mathrm{CI}) \\
\text { male: female adjusted odds ratio }(95 \% \mathrm{CI})\end{array}$ & $\begin{array}{l}40.4(37.0) \\
22.4 \\
10.7(10.0) \\
13.4(13.0) \\
5.9\end{array}$ & $\begin{array}{l}39.6(38.0) \\
31.6 \\
10.2(10.0) \\
13.1(12.0) \\
9.1 \\
0.65(0.31 \text { to } 1.34) \\
0.53(0.24 \text { to } 1.18)\end{array}$ & $\begin{array}{l}0.27 \dagger \\
0.01^{*} \\
0.75 \dagger \\
0.59 \dagger \\
0.16^{*}\end{array}$ \\
\hline Self poisoning & $\begin{array}{l}\text { Mean (median) age } \\
\% \text { PMH } \\
\text { Mean (median) APS } \\
\text { Mean (median) APACHE II } \\
\% \text { Hospital mortality } \\
\text { male: female crude odds ratio }(95 \% \mathrm{CI}) \\
\text { male: female adjusted odds ratio }(95 \% \mathrm{CI})\end{array}$ & $\begin{array}{l}34.9(33.0) \\
1.1 \\
13.4(13.0) \\
14.1(14.0) \\
8.5\end{array}$ & $\begin{array}{l}37.2(35.0) \\
1.6 \\
13.1(13.0) \\
14.1(14.0) \\
8.6 \\
1.12(0.75 \text { to } 1.67) \\
1.01(0.65 \text { to } 1.56)\end{array}$ & $\begin{array}{l}0.002 \dagger \\
0.43^{*} \\
0.39 \dagger \\
0.99 \dagger \\
0.97^{*}\end{array}$ \\
\hline
\end{tabular}

*Based on $\chi^{2}$ test; †based on $t$ test. APS, acute physiology score; PMH, past medical history; APACHE II, APACHE II score.

score, but men had a higher hospital mortality than women (table 2). After adjustment for case mix, men still had a significantly higher likelihood of hospital death (adjusted OR $1.28,95 \%$ CI 1.06 to 1.54 ) than women.

\section{Chronic obstructive airways disease}

There were no significant gender differences with respect to age, acute physiology score, past medical history, APACHE II score, or mortality (table 2 ).

\section{Asthma}

There were no significant gender differences with respect to age, acute physiology score, past medical history, APACHE II score, or mortality (table 2 ).

\section{Self poisoning}

Men were significantly younger than women but there were no significant gender differences with respect to acute physiology score, past medical history, APACHE II score, or mortality (table 2).

\section{Ventricular failure}

There were no significant gender differences with respect to age, acute physiology score, past medical history, or APACHE II score, but men had a higher hospital mortality than women (table 3). After adjustment for case mix, men still had a greater likelihood of hospital death than women, but this was not significant at the $5 \%$ level (adjusted OR 1.27, 95\% CI 0.91 to 1.75 ).

\section{Cardiac arrhythmia}

Male patients were significantly younger than their female counterparts, but there were no gender differences in acute physiology score, past medical history, APACHE II score, or the likelihood of hospital death (table 3 ).

\section{Primary brain injury}

There were no significant gender differences with respect to age, acute physiology score, past medical history, or APACHE II score (which was asymmetrically distributed towards lower scores) (table 3). However, men had a lower rate of hospital death than women. Men were still found to be less likely to suffer hospital death after adjustment for APACHE II score, though this difference was no longer significant at the $5 \%$ level (adjusted OR $0.62,95 \%$ CI 0.38 to 1.01 ).

\section{Seizures}

Men were older than women (table 3). There were no significant gender differences with respect to acute physiology score, past medical history, APACHE II score, or mortality.

\section{Neurological bleeding}

Male patients were significantly younger than female patients and were less severely ill according to the acute physiology score (table 3). There were no significant gender differences in past medical history. The APACHE II score was lower in men than women and men were less likely to die in hospital. Men were still found to be less likely to suffer hospital death after adjustment for APACHE II score, though this was no longer significant at the 5\% level (adjusted OR 0.71, 95\% CI 0.48 to 1.08). 


\begin{tabular}{|c|c|c|c|c|}
\hline Category & Measure & Men & Women & Difference \\
\hline Ventricular failure & $\begin{array}{l}\text { Mean (median) age } \\
\% \text { PMH } \\
\text { Mean (median) APS } \\
\text { Mean (median) APACHE II } \\
\% \text { Hospital mortality } \\
\text { male: female crude odds ratio }(95 \% \mathrm{CI}) \\
\text { male: female adjusted odds ratio }(95 \% \mathrm{CI})\end{array}$ & $\begin{array}{l}68.1(71.0) \\
24.5 \\
15.5(15.0) \\
21.0(21.0) \\
55.9\end{array}$ & $\begin{array}{l}68.8(71.0) \\
24.4 \\
14.9(14.0) \\
20.6(20.0) \\
48.6 \\
1.30(0.95 \text { to } 1.75) \\
1.27(0.91 \text { to } 1.75)\end{array}$ & $\begin{array}{l}0.43 \dagger \\
0.97 * \\
0.20 \dagger \\
0.36 \dagger \\
0.03^{*}\end{array}$ \\
\hline Cardiac arrhythmia & $\begin{array}{l}\text { Mean (median) age } \\
\% \text { PMH } \\
\text { Mean (median) APS } \\
\text { Mean (median) APACHE II } \\
\% \text { Hospital mortality } \\
\text { male: female crude odds ratio }(95 \% \mathrm{CI}) \\
\text { male: female adjusted odds ratio }(95 \% \mathrm{CI})\end{array}$ & $\begin{array}{l}63.9(66.0) \\
13.6 \\
14.7(13.0) \\
19.4(18.0) \\
41.4\end{array}$ & $\begin{array}{l}68.2(70.0) \\
14.9 \\
14.4(13.0) \\
19.6(19.0) \\
45.9 \\
0.94(0.67 \text { to } 1.32) \\
0.92(0.62 \text { to } 1.35)\end{array}$ & $\begin{array}{l}<0.001 \dagger \\
0.59 * \\
0.73 \dagger \\
0.82 \dagger \\
0.18^{*}\end{array}$ \\
\hline Primary brain injury & $\begin{array}{l}\text { Mean (median) age } \\
\% \text { PMH } \\
\text { Mean (median) APS } \\
\text { Mean (median) APACHE II } \\
\% \text { Hospital mortality } \\
\text { male: female crude odds ratio }(95 \% \mathrm{CI}) \\
\text { male: female adjusted odds ratio }(95 \% \mathrm{CI})\end{array}$ & $\begin{array}{l}34.1(29.0) \\
2.2 \\
12.2(10.0) \\
13.4(12.0) \\
28.7\end{array}$ & $\begin{array}{l}35.7(30.0) \\
1.0 \\
13.0(11.0) \\
14.8(13.0) \\
39.2 \\
0.58(0.39 \text { to } 0.87) \\
(0.62(0.38 \text { to } 1.01)\end{array}$ & $\begin{array}{l}0.90 \ddagger \\
0.29 * \\
0.27 \dagger \\
0.13 \ddagger \\
0.004^{*}\end{array}$ \\
\hline Seizures & $\begin{array}{l}\text { Mean (median) age } \\
\% \text { PMH } \\
\text { Mean (median) APS } \\
\text { Mean (median) APACHE II } \\
\% \text { Hospital mortality } \\
\text { male: female crude odds ratio }(95 \% \mathrm{CI}) \\
\text { male: female adjusted odds ratio }(95 \% \mathrm{CI})\end{array}$ & $\begin{array}{l}39.3(39.0) \\
8.9 \\
15.1(14.0) \\
19.7(19.0) \\
11.5\end{array}$ & $\begin{array}{l}34.7(32.0) \\
10.1 \\
11.3(10.0) \\
13.0(12.0) \\
11.0 \\
0.94(0.53 \text { to } 1.67) \\
0.76(0.41 \text { to } 1.41)\end{array}$ & $\begin{array}{l}0.01 \dagger \\
0.63^{*} \\
0.21 \dagger \\
0.1 \dagger \\
0.85^{*}\end{array}$ \\
\hline Neurological bleeding & $\begin{array}{l}\text { Mean (median) age } \\
\% \text { PMH } \\
\text { Mean [median) APS } \\
\text { Mean (median) APACHE II } \\
\% \text { Hospital mortality } \\
\text { male: female crude odds ratio }(95 \% \mathrm{CI}) \\
\text { male: female adjusted odds ratio }(95 \% \mathrm{CI})\end{array}$ & $\begin{array}{l}46.7(49.0) \\
2.7 \\
15.0(14.0) \\
17.1(16.0) \\
59.4\end{array}$ & $\begin{array}{l}51.0(52.0) \\
3.5 \\
16.4(15.0) \\
18.9(17.0) \\
71.2 \\
0.64(0.44 \text { to } 0.93) \\
0.71(0.48 \text { to } 1.08)\end{array}$ & $\begin{array}{l}<0.001 \dagger \\
0.53^{*} \\
0.02 \dagger \\
0.005 \dagger \\
<0.001 *\end{array}$ \\
\hline
\end{tabular}

* Based on $\chi^{2}$ test; tbased on $t$ test; łbased on Mann-Whitney U test. APS, acute physiology score; PMH, past medical history; APACHE II, APACHE II score.

\section{DISCUSSION \\ Study findings}

This study compared the case mix and vital status at hospital discharge of men and women admitted to ICU for 10 different clinical categories. For five categories (cardiac arrhythmia, chronic obstructive airways disease, asthma, self poisoning, and seizures) there were no gender differences either in case mix or in mortality (that is, no evidence of gender bias). The hypothesis that patients who subscribed to the gender norm for a condition would exhibit less severe illness on admission to ICU compared with those who did not, was not demonstrated for self poisoning, asthma or chronic obstructive airways disease.

There was some evidence that horizontal and vertical equity was not achieved for patients with myocardial infarction and with neurological bleeding. Male admissions had lower APACHE II scores than women. This suggests that disease severity admission criteria for women were more stringent than for men and horizontal inequity occurred. Male admissions were also less likely to die after adjusting for APACHE II score, although these results did not reach statistical significance at the $5 \%$ level. This suggests the presence of vertical inequity because the unequal use of intensive care could not be explained by an unequal need for such care.

In addition, vertical equity was not achieved for patients with primary brain injury, pneumonia or ventricular failure. Although there were no gender differences in the case mix of admissions to intensive care for these groups of patients (that is, no horizontal inequity), male brain injury patients were less likely to die than female patients (a finding of borderline statistical significance) - whereas male patients with pneumonia were more likely to die in hospital than female admissions. In addition, men with ventricular failure had a $27 \%$ greater odds of hospital mortality than women. This suggests gender differences in the need for care, and that female patients with primary brain injury and male patients with pneumonia or ventricular failure may not receive the care they need.

\section{Methodological issues}

The strengths of this study are that a broad view of gender differences in specialist health care use for a wide range of diseases could be obtained by examining prospectively collected national data. Secondly, the units involved include both tertiary and secondary hospitals from across England, Wales, and Northern Ireland, and so the results are likely to be representative of care nationally. Thirdly, quality control mechanisms used by ICNARC ensure that the data collected for incorporation into the score are standard, complete, and accurate. Finally, while we can never be certain that all potential risk factors have been taken into account, the APACHE II score has been extensively validated in the UK and shown to be highly associated with hospital mortality. ${ }^{10}$

A limitation of this study was the inability to examine equity in the entire population at risk. Its cross sectional nature meant that data on the clinical need of all patients admitted to hospital (including those not admitted to ICU) with the conditions studied were not available. 


\section{Key points}

- Gender bias in the provision of specialist care has been examined for patients with coronary artery disease, but other conditions have barely been investigated.

- It can result in either over-treatment or under-treatment. Intensive care patients provide an appropriate sample to test whether bias occurs across a range of conditions.

- This study showed, for the first time, inequitable use of intensive care for patients with certain conditions. The findings may be attributable to the application of stereotypical assumptions to patients with myocardial infarction, neurological bleeding, and primary brain injury. Biological differences may explain the findings for patients with pneumonia.

- These results should stimulate further investigation of the existence of gender bias, and its causes.

Secondly, the effectiveness of intensive care has been subject to little evaluation. ${ }^{10}$

It was therefore necessary to assume that intensive care is equally beneficial to men and women, and so should be available to them purely on the basis of their clinical need.

Thirdly, it should be emphasised that the results of this study only relate to the conditions studied and it should not be assumed that they can be generalised to other conditions treated in intensive care, or to patients admitted via the operating theatre.

Fourthly, a potential limitation of the APACHE II score should be considered. Reported gender differences in APACHE II scores (which are based on the first 24 hours in ICU) could be secondary to a systematic tendency to stabilise either men or women before admission. However, examination of the number of days in hospital immediately prior to admission to intensive care showed no gender differences, suggesting that lead time bias did not occur. However, the physiological findings may be affected by the treatment received in the ICU which may, in turn, be influenced systematically by patient gender. This requires further investigation.

For two conditions, asthma and primary brain injury, the power available to detect real differences was low so the lack of statistical significant differences does not preclude the existence of real, clinically significant differences. Thus, although male patients with brain injury had lower APACHE II scores than women, the difference did not reach significance at the 5\% level. A larger, more powerful study may have demonstrated horizontal inequity in favour of men.

Finally, the study had less power to detect important differences in hospital mortality than it did to detect gender differences in APACHE II score for two reasons. Firstly, mortality was, in some categories (asthma and self poisoning), an uncommon event. Secondly, categorical outcome measures such as mortality require larger sample sizes than continuous outcome measures to achieve the same power. This may explain why the adjusted odds ratios for myocardial infarction, ventricular failure, primary brain injury, and neurological bleeding did not reach significance despite being quite pronounced. The statistical significance of these results should therefore be treated with caution.

\section{Explanations}

The horizontal inequity found for patients with myocardial infarction and neurological bleeding implies more restrictive admission criteria for women. This could be explained by the "gender norm" hypothesis. Thus, if the majority of admissions with neurological bleeding are attributable to traumatic head injury (a condition commoner in men ${ }^{13}$ ), and if clinicians associate trauma with men, then the lower APACHE II scores found in male patients could be explained in terms of the "gender norms" hypothesis.

\section{Policy implications}

This study showed, for the first time, inequitable use of intensive care for patients with certain conditions. If further research confirms these findings and clarifies the extent to which patient and health service factors, including gender bias, influence these results, then there will be important policy implications. Findings of gender inequities in health care use would be expected to prompt changes in NHS policy in order to safeguard the fundamental principle that health care should be provided solely on the basis of clinical need. Such changes may include ensuring that gender differences in health care use are monitored, and developing transparent criteria for deciding the level of clinical need at which intervention should occur.

The "gender norm" hypothesis may also explain the findings for patients admitted with primary brain injury. More than three quarters of the admissions were male. If this predominance is expected by clinicians because primary brain injury, commonly a result of trauma, ${ }^{13}$ is seen as a "male" condition, then the "gender norms" hypothesis would suggest that men would have lower APACHE II scores and be less likely to die in hospital than women, because they would both be more readily admitted to ICU and more readily treated. A lower likelihood of death among men was indeed found in this study. Further examination of this hypothesis requires analysis of disaggregated data on each of the diagnoses within these disease categories. The extent to which clinicians associate the component conditions with gender also needs to be examined, as does the diagnosis, management and outcome of patients in these categories who are not admitted to ICU.

The reason for the higher mortality among male pneumonia patients, who were remarkably similar to female patients with respect to case mix, is unclear. It may be that there are biological differences between male and female pneumonia patients that were unaccounted for or not known.

Gender bias against male ventricular failure patients could explain the higher male mortality. This is an intriguing possibility in view of the fact that ventricular failure is most commonly secondary to hypertension and coronary artery disease. ${ }^{14}$ Where gender bias has been reported in specialist health care for coronary artery disease patients, it is mostly found to be in favour of men. ${ }^{15}$ It may be that two different populations are being studied. It is known that those patients who are admitted with a primary diagnosis of myocardial infarction and who develop ventricular failure are deemed to have a need for health care (such as revascularisation)..$^{16}$ It could be that such patients are viewed differently to admissions with a primary diagnosis of ventricular failure. Ventricular failure patients are older and have higher APACHE II scores than myocardial infarction patients (table 2). They also have higher mortality. It is conceivable that ventricular failure patients are thought to have a poor prognosis and while they may have a need for health, they may not be seen to have a need for health care. These patients may therefore be treated differently to acute cardiac patients. Why this should result in a bias against men requires further study.

\section{Conclusions}

This study demonstrated, for the first time, inequitable use of intensive care in favour of male patients with myocardial infarction, neurological bleeding, and primary brain injury; and in favour of female patients with pneumonia and ventricular failure. The "gender norms" hypothesis may explain the findings for patients with myocardial infarction, neurological bleeding, and primary brain injury. However, no gender difference was found for other categories, which suggests that 
a general bias for or against men or women does not occur. When gender bias does occur, it represents the inequitable use of health care that does not conform to the aspirations of the British National Health Service. In practical terms, gender bias can result in either over-treatment in the favoured group or under-treatment in the neglected group. Neither group is well served. In view of the dearth of research into gender differences in the use of specialist services for many conditions these results should stimulate further study both to reexamine the findings reported here and to investigate further the influence of patient and health service factors, including gender bias, in producing these results.

\section{ACKNOWLEDGEMENTS}

We thank everyone in the ICUs participating in the Case Mix Programme and those responsible for local funding.

\section{Authors' affiliations}

R Raine, N Black, Health Services Research Unit, London School of Hygiene and Tropical Medicine, London, UK

C Goldfrad, K Rowan, ICNARC, London, UK

Funding: Rosalind Raine was funded by the MRC/NHSE London Region.

Conflicts of interest: none.

\section{REFERENCES}

1 Kumar P, Clark M. Clinical medicine. London: Balliere Tindall, 1999.
2 Smith G, Nielson M. A B C of Intensive Care: criteria for admission. BM 2000;318:1544-7.

3 Raine R. Does gender bias exist in the use of specialist health care? J Health Serv Res Policy 2000;5:237-49.

4 Healy B. The Yentl syndrome. NEJM 1991;325:274-5.

5 Farmer R, Miller D. Lecture notes on epidemiology and community medicine. London: Blackwell Scientific, 1988.

6 Mooney G. And now for vertical equity? Some concerns arising from aboriginal health in Australia. Health Econ 1996;5:99-103.

7 Rowan K, Black N. A bottom-up approach to performance indicators through clinician networks. Health Care UK 2000:42-6.

8 Goldfrad C, Rowan K. Consequences of discharges from intensive care at night. Lancet 2000;355: $1138-42$.

9 ICNARC. ICNARC case mix programme data set specification, version 2.0. London: ICNARC, 1999.

10 Rowan K. Risk adjustment for intensive care outcomes. In: Goldhill D, Withington S, eds. Textbook of intensive care. London: Chapman and Hall, 1997.

11 Lerner D, Kannel W. Patterns of coronary heart disease morbidity and mortality in the sexes; a 26 year follow-up of the Framingham population. Am Heart J 1986;111:383-90.

12 McLoone P, Crombie I. Hospitalisation for deliberate self-poisoning in Scotland from 1981 to 1993: trends in rates and types of drugs used. $\mathrm{Br}$ J Psychiatry 1996;169:81-5.

13 Guerrero J, Thurman D, Sniezek J. Emergency department visits associated with traumatic brain injury; United States, 1995-1996. Brain Inj 2000; 14:181-6.

14 Kannel W. Vital epidemiologic clues in heart failure. J Clin Epidemiol 2000;53:229-35.

15 Jackson G. Coronary artery disease and women. BM 1994:309:226-7.

16 American College of Cardiology/American Heart Association Task Force Report. Guidelines and Indications for Coronary Artery Bypass Graft Surgery. J Am Coll Cardiol 1991;17:543-89.

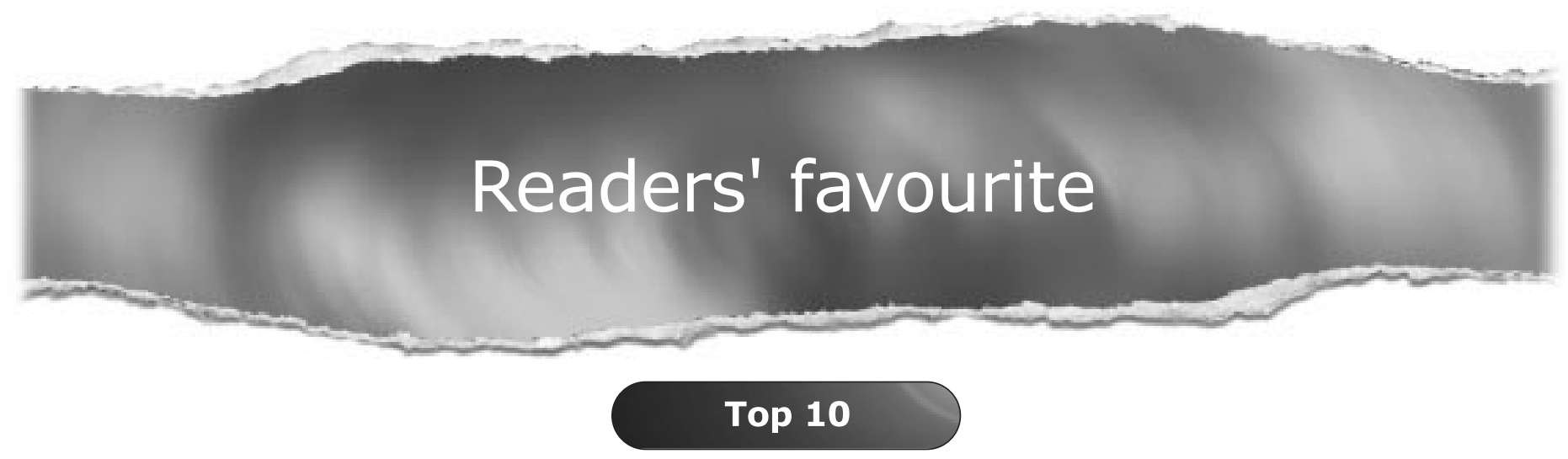

Click on the "Top 10" button on the homepage to see which are the best read articles each month

www.jech.com 